\title{
Encoding the Odor of Cigarette Smoke
}

\author{
${ }^{1}$ Timothy S. McClintock, ${ }^{1}$ Naazneen Khan, ${ }^{1}$ Yelena Alimova, ${ }^{1}$ Madeline Aulisio, ${ }^{2}$ Dong Y. Han, ${ }^{3}$ \\ and Patrick Breheny ${ }^{4}$ \\ ${ }^{1}$ Department of Physiology, University of Kentucky, Lexington, Kentucky 40536, ${ }^{2}$ College of Public Health, University of Kentucky, Lexington, \\ Kentucky 40536, ${ }^{3}$ Department of Neurology, University of Kentucky, Lexington, Kentucky 40536, and ${ }^{4}$ Department of Biostatistics, University of \\ Iowa, Iowa City, Iowa 52242
}

The encoding of odors is believed to begin as a combinatorial code consisting of distinct patterns of responses from odorant receptors (ORs), trace-amine associated receptors (TAARs), or both. To determine how specific response patterns arise requires detecting patterns in vivo and understanding how the components of an odor, which are nearly always mixtures of odorants, give rise to parts of the pattern. Cigarette smoke, a common and clinically relevant odor consisting of $>400$ odorants, evokes responses from 144 ORs and 3 TAARs in freely behaving male and female mice, the first example of in vivo responses of both ORs and TAARs to an odor. As expected, a simplified artificial mimic of cigarette smoke odor tested at low concentration to identify highly sensitive receptors evokes responses from four ORs, all also responsive to cigarette smoke. Human subjects of either sex identify 1-pentanethiol as the odorant most critical for perception of the artificial mimic; and in mice the OR response patterns to these two odors are significantly similar. Fifty-eight ORs respond to the headspace above 25\% 1-pentanethiol, including 9 ORs responsive to cigarette smoke. The response patterns to both cigarette smoke and 1-pentanethiol have strongly responsive ORs spread widely across OR sequence diversity, consistent with most other combinatorial codes previously measured in vivo. The encoding of cigarette smoke is accomplished by a broad receptor response pattern, and 1-pentanethiol is responsible for a small subset of the responsive ORs in this combinatorial code.

Key words: GPCR; olfaction; perception; sensory; physiology; smoking

Significance Statement

Complex odors are usually perceived as distinct odor objects. Cigarette smoke is the first complex odor whose in vivo receptor response pattern has been measured. It is also the first pattern shown to include responses from both odorant receptors and trace-amine associated receptors, confirming that the encoding of complex odors can be enriched by signals coming through both families of receptors. Measures of human perception and mouse receptor physiology agree that 1-pentanethiol is a critical component of a simplified odorant mixture designed to mimic cigarette smoke odor. Its receptor response pattern helps to link those of the artificial mimic and real cigarette smoke, consistent with expectations about perceptual similarity arising from shared elements in receptor response patterns.

\section{Introduction}

Natural odors are mixtures of many species of volatile chemicals, known as odorants. Odors are initially encoded as "combinatorial codes" consisting of the response patterns of receptor proteins located in the cilia of olfactory sensory neurons (OSNs) (Malnic et al., 1999; Nara et al., 2011). The majority of odorantresponsive receptors are the odorant receptors (ORs), which

\footnotetext{
Received May 11, 2020; revised July 23, 2020; accepted Aug. 9, 2020.

Author contributions: T.S.M., D.Y.H., and P.B. designed research; T.S.M., N.K., Y.A., and M.A. performed research; T.S.M. and P.B. analyzed data; T.S.M. wrote the paper; D.Y.H. and P.B. edited the paper.

This work was supported by National Institutes of Health R01DC014423; University of Kentucky Vice President for Research IRC program award to T.S.M.

T.S.M. has an equity interest in a company based on technologies used to measure responses to odors. The remaining authors declare no competing financial interests.

Correspondence should be addressed to Timothy S. McClintock at mcclint@uky.edu.

https://doi.org/10.1523/JNEUROSCl.1144-20.2020

Copyright $\odot 2020$ the authors
}

number in the hundreds in most mammals and $\sim 1100$ in mice (Buck and Axel, 1991; Niimura et al., 2014). Also important for odor detection are trace amine-associated receptors (TAARs). In mice, all but one of the 15 TAARs are expressed in OSNs where they are important for detecting volatile amines (Liberles and Buck, 2006; Ferrero et al., 2012; Johnson et al., 2012; Dewan et al., 2018). Each mouse OSN expresses just one allele of one OR or TAAR gene (Chess et al., 1994; Mombaerts, 2004; Liberles, 2015). This maximizes the distinctiveness of each OSN's response and allows each olfactory bulb glomerulus to be innervated only by axons of OSNs expressing the same OR or TAAR (Mombaerts et al., 1996), so that receptor response patterns can be represented faithfully in spatiotemporal patterns of glomerular activity.

We understand little about how OR and TAAR response patterns contribute to perception. For example, are response patterns similar for odors that have similar percepts? Are response 
patterns to complex odors necessarily broad, or do antagonistic interactions between odorants at receptors (de March et al., 2020) cause complex odors to have narrow response patterns? Are response patterns determined by only a few of the odorants in a complex odor? Are there core sets of odorants and receptors that determine perception of odors? This idea of core sets of receptors for percepts is consistent with the ability of chemists to mimic the percept evoked by a complex odor with just a few of its odorants (Tamura et al., 2008) and with concentrationinvariant perception of odors (Friedrich and Korsching, 1997; Rubin and Katz, 1999; Meister and Bonhoeffer, 2001; Wachowiak and Cohen, 2001; Fried et al., 2002; Bozza et al., 2004; Storace and Cohen, 2017; Bolding and Franks, 2018). In this study, we investigated the receptor response pattern of a complex odor and the contribution of one of its odorants.

To focus our efforts on an odor significant to humans, we investigated cigarette smoke. Not only is it a common odor, it has clinical importance. In smokers and reformed smokers, the odor of cigarette smoke increases the desire to smoke (Cortese et al., 2015a,b). The link between the olfactory system and the brain's reward circuit has become hijacked by nicotine's activation of the reward circuit during exposure to cigarette smoke (Picciotto and Mineur, 2014; Balfour, 2015). This physiological response contributes to the difficulty smokers encounter when they attempt smoking cessation (Halpern et al., 2018; Hajek et al., 2019). Exposing freely behaving mice to cigarette smoke evokes a broad response pattern containing both ORs and TAARs. A small subset of these ORs comprise the response pattern to a low concentration of an artificial mimic of cigarette smoke odor. One of the odorants in this mimic, 1-pentanethiol, is especially important for human perception of artificial cigarette smoke odor and drives responses from several of the mouse ORs responsive to artificial cigarette smoke and to real cigarette smoke.

\section{Materials and Methods}

Materials. Odorants were obtained at the highest purity available. Indole and butyric acid were kind gifts from Firmenich SA. Thiophene and 1,3-cyclohexadiene were purchased from Alfa Aesar. All other odorants were purchased from Sigma Millipore. An artificial cigarette smoke odor designed to mimic the odor of cigarette smoke (Dravnieks et al., 1975) was formulated by mixing 26 odorants at the proportions shown in Table 1. This artificial cigarette smoke odor should not be confused with commercial products mimicking cigarettes or cigarette smoke. Such products are designed to mimic certain properties of cigarettes, such as visual appearance, but not their odor.

Human subject testing. The procedures used for human subjects were approved by the University of Kentucky Institutional Review Board. Male and female volunteers ages 18-50 from the Lexington, KY consented in writing to participate in this study. Smokers, pregnant women, persons with active sinus infections, persons with a history of smell or taste deficits, persons suffering from fragrance allergies or chemical sensitivity, persons with a history of migraines headaches, and persons with a diagnosed neurologic disorder were excluded.

Tests of similarity between artificial cigarette smoke odor and odorant depletion mixtures lacking one of the odorants in artificial cigarette smoke odor were done by 18 consenting subjects: 10 females and 8 males. A total of 27 odor mixtures were prepared: the full artificial cigarette smoke odor mixture and 26 mixtures, each lacking one of the 26 odorants in the full mixtures. The mixtures were prepared fresh on the day of testing and absorbed into cotton balls sealed in brown glass vials. Subjects were seated in front of Movex fume capture hoods used to prevent odors from filling the testing room and causing adaptation. Subjects were first asked to familiarize themselves with the artificial cigarette smoke odor. Using intervals of at least $1 \mathrm{~min}$ between odor
Table 1. Formulation of artificial cigarette smoke odor ${ }^{\mathrm{a}}$

\begin{tabular}{lcc}
\hline \multicolumn{1}{c}{ Odorant } & CAS \# & Volume $(\mu \mathrm{ll})$ \\
\hline Pyridine & $110-86-1$ & 5 \\
Nicotinaldehyde & $500-22-1$ & 15 \\
5-Ethyl-2-methylpyridine & $104-90-5$ & 30 \\
2-Vinylpyridine & $100-69-6$ & 5 \\
4-Pyridinecarbonitrile & $100-48-1$ & $5 \mathrm{mg}$ \\
Methyl isonicotinate & $2459-09-8$ & 30 \\
2-Ethyl-3-methylpyrazine & $15707-23-0$ & 20 \\
2-Methoxy-3,5-methylpyrazine & $93905-03-4$ & 4 \\
2-Methoxyphenol & $90-05-1$ & 15 \\
0-Cresol & $95-48-7$ & 5 \\
5-Methylfurfural & $620-02-0$ & 15 \\
2,5-Dimethylpyrrole & $625-84-3$ & 30 \\
Phenylacetylene & $536-74-3$ & 15 \\
Isoprene & $78-79-5$ & 15 \\
1,3-Cyclohexadiene & $592-57-4$ & 5 \\
Indene & $95-13-6$ & 15 \\
Allylbenzene & $300-57-2$ & 15 \\
2,3-Butanedione & $431-03-8$ & 10 \\
Thiophene & $110-02-1$ & 5 \\
1-Pentanethiol & $110-66-7$ & 2.5 \\
Allylamine & $107-11-9$ & 2.5 \\
1-Aminopentane & $110-58-7$ & 2.5 \\
Butyric acid & $107-92-6$ & 10 \\
1-Hexanal & $66-25-1$ & 5 \\
Trans,trans-2,4-Hexadienal & $142-83-6$ & 5 \\
Indole & $120-72-9$ & $5 \mathrm{mg}$ \\
\hline
\end{tabular}

andole and 4-pyridinecarbonitrile were dissolved at $0.5 \mathrm{mg} / \mu \mathrm{l}$ in DMSO and $10 \mu \mathrm{l}$ of each added to the mixture.

sampling, each subject was given each of the depleted odorant mixtures in an order uniquely randomized for each subject. Subjects scored these mixtures using a method modeled after those of Keller and Vosshall (2016). The similarity of each odorant-deficient mixture to the full mixture was scored on a scale of 0 to 100 , with 0 representing no similarity and 100 representing identical sensations.

Another 27 consenting subjects, 17 females and 10 males, were used to test whether individual odorants were discriminably different from the artificial cigarette smoke odor mixture. Subjects were seated in front of Movex fume capture hoods and asked to familiarize themselves with the odor of the full artificial cigarette smoke odor mixture. Using intervals of at least $1 \mathrm{~min}$ between odorants, each subject was given an odorant to sample and asked to rate its similarity to the full synthetic cigarette smoke odor mixture on a scale of 0 to 100 . Subjects then also rated their perception of the pleasantness of each odorant, again using a scale of 0 to 100 , with 0 being the most unpleasant odor imaginable and 100 being the most pleasant odor they could imagine. Each subject sampled the odors in a unique random order.

Detection of OR and TAAR responses in live mice. In this assay, odor stimulated expression of GFP from the activity-dependent S100A5 gene locus in the S100a5-tauGFP mouse (The Jackson Laboratory, stock $\# 006709$ ). The S100a5-tauGFP ${ }^{(+)}$mice used for this project are from a stock back-crossed for 10 generations against C57BL/6J. Both sexes of mice, ages 7-12 weeks, were used. All procedures with mice were done according to protocols approved by the Institutional Animal Care and Use Committee of the University of Kentucky. $\mathrm{GFP}^{+}$and $\mathrm{GFP}^{-}$cell samples were collected by FACS of dissociated olfactory mucosae of heterozygous S100a5-tauGFP mice after stimulation with odor or with clean air. Because each OSN only expresses a single OR or TAAR gene, the OR and TAAR mRNAs specifically enriched in samples from mice stimulated with odorant but not in samples from mice stimulated with clean air must encode receptors responsive to the odorants tested. The reliability of this assay of receptor responses to odorants in freely behaving mice has been confirmed by in vitro studies of individual OR responses expressed in cultured cells (McClintock et al., 2014; de March et al., 2020). 
Each mouse was housed individually in specially designed, heated $\left(27^{\circ} \mathrm{C}\right)$ Plexiglas chambers $\left(700 \mathrm{~cm}^{3}\right)$ under a flow of $3.1 \mathrm{l} / \mathrm{min}$ of filtered air for $40 \mathrm{~h}$ to allow degradation of GFP evoked by prior odor exposure. Over the last $16 \mathrm{~h}$ of this $40 \mathrm{~h}$ period, the mice were stimulated by activating computer-controlled solenoid valves that divert the flow of filtered air to flush the headspace from a $50 \mathrm{ml}$ Delrin vial containing $5 \mathrm{ml}$ of either the DMSO vehicle or 25\% 1-pentanethiol in DMSO. GFP fluorescence in an activated OSN peaks at 6-8 $\mathrm{h}$ after the onset of stimulation and because GFP has a 26 h half-life (Corish and Tyler-Smith, 1999) once an OSN responds to an odor it is marked for longer than the duration of the experiment. To stimulate with cigarette smoke, a $590 \mathrm{~cm}^{3}$ Plexiglas cylinder closed at the bottom and containing a lit 1R6F Research Cigarette (produced by the Center for Tobacco Reference Products at the University of Kentucky) was secured against the wire mesh bottom of each mouse chamber for $1 \mathrm{~min}$ and the airflow to each chamber was discontinued, allowing smoke to enter the mouse chamber. For control mice, the same cylinder was used but it contained no cigarette. This procedure began $16 \mathrm{~h}$ before death of the mice and was done 6 times with $30 \mathrm{~min}$ between stimulations. This same procedure was used for stimulation with artificial cigarette smoke odor, using $0.6 \mathrm{ml}$ of the artificial cigarette smoke odor source mixture or a nonvolatile vehicle (DMSO) soaked into a cotton ball as the stimuli. At the completion of stimulation, olfactory mucosae were dissected and cells dissociated in a procedure involving papain, trypsin, deoxyribonuclease, and low calcium saline as described previously (Yu et al., 2005; Sammeta et al., 2007). Cells from three identically treated mice were pooled, and FACS was performed in the University of Kentucky Flow Cytometry and Cell Sorting Facility using an iCyt Synergy cell sorting system to collect $\mathrm{GFP}^{+}$and $\mathrm{GFP}^{-}$cell samples. Total RNA was isolated using the QIAGEN RNeasy Micro kit (catalog \#74004). RNA quantity was measured using Affymetrix Mouse Clariom S arrays in the University of Kentucky Microarray Facility. The microarray data are available in Gene Expression Omnibus under accession number GSE146418. Data were initially processed using Affymetrix GeneChip Command Console software to generate globally normalized quantities for each gene transcript cluster. Additional processing to generate $\mathrm{GFP}^{+} / \mathrm{GFP}^{-}$ratios from the microarray signal intensities was done in Microsoft Excel. These $\mathrm{GFP}^{+}$/ $\mathrm{GFP}^{-}$enrichment ratios are equivalent to fold differences and help to normalize effect across the different abundances of mRNAs and across differences in constitutive activity of ORs and TAARs. The median signal intensity of 135 mature OSN-specific mRNAs (Nickell et al., 2012) was used to adjust for differences in the amount of mature OSNs across samples.

Experimental design and statistical analysis. Both human subject experiments were shared control designs where each condition was compared with a single common control, which in both cases was the full artificial cigarette smoke odor mixture. The test administrator was blind to the identities of the odors, each being identified only by a unique code on the odor vial. A different person performed statistical analyses of these data and was also blind to the identity of the odorants represented by the unique codes. Rating scores for each individual were converted to rankings and analyzed by estimation statistics for effect size using a shared control model for mean differences (http://www.estimationstats. com/\#/analyze/shared-control). Effect sizes and CIs were calculated and are displayed. The $p$ values reported for the effect sizes derive from Welch's unequal variance $t$ test (Welch, 1947).

The in vivo assay design was a paired comparison of a group of 3 mice of either or both sexes exposed to odor to a sex- and age-matched group of 3 mice simultaneously exposed to filtered air, replicated 4 times. For analysis of these data, we used a Bayesian hierarchical model to obtain normalized measures of odorant effect, accounting for four sources of variation: basal receptor effect, odorant effect, nonspecific effect (change in both odorant and vehicle control), and random measurement error. For each odorant effect, the posterior mean divided by the posterior SD provides a measure ( $Z$ statistic) that is approximately normally distributed. Local false discovery rates (FDRs) (Efron, 2008; Stephens, 2017) were used to estimate the probability that each receptor was responsive to the odorant using a uniform mixture model. Based on the identification of responses from ORs whose agonists were identified using independent methods, a 15\% FDR was found to be a suitable level of risk for the identification of activated receptors (McClintock et al., 2014). Data for each receptor mRNA are reported as the $\mathrm{GFP}^{+} / \mathrm{GFP}^{-}$ratio fold differences for odor-stimulated mice and for control mice. The overall response measure (delta) is the $\mathrm{GFP}^{+} / \mathrm{GFP}^{-}$ratio fold difference for odorstimulated mice divided by the $\mathrm{GFP}^{+} / \mathrm{GFP}^{-}$ratio fold difference for control mice. Responsive ORs show a large delta value because their mRNAs increase in the $\mathrm{GFP}^{+}$sample while simultaneously decreasing in the $\mathrm{GFP}^{-}$ samples from odor-stimulated mice, but not in samples from control mice. Phylogenetic tree plots of OR sequence distance were generated in R. Dendrograms of the relationships between OR family size and the fraction of responsive ORs per family were generated using hierarchical clustering (Euclidean distance) functions from the R cluster library.

To compare receptor response patterns, we performed cluster analysis based on the following distance metric. To account for changes in overall magnitude of response, fold change differences on the log scale were quantile-normalized across experiments. A soft threshold was then applied so that only changes larger than twofold enrichment contributed to the distance. Euclidean distances between odor responses were then calculated. To assess whether odor responses were significantly similar, permutation tests were applied, forming a null distribution of odor distances by drawing 10,000 random permutations of the responses. This was done following the quantile normalization step, so that the distribution of responses was the same across all odorant response experiments. The permutation testing was implemented in R 4.0.2 (www.R-project.org).

\section{Results}

\section{ORs and TAARs responsive to cigarette smoke}

Cigarette smoke is highly complex, consisting of particulate matter, gases, and a wide variety of organic chemicals produced during the incomplete combustion of cigarettes (Rodgman and Perfetti, 2013). Among these are $>400$ structurally diverse volatile organic chemicals, known as odorants, making it one of the most complex odors known. Its complexity, which includes volatile amines, predicts that a broad array of ORs and TAARs would respond to it. To test this prediction, we used an in vivo assay that allows us to identify receptors responsive to any odor presented to freely behaving mice (McClintock et al., 2014). Expression of GFP from the S100a5 gene locus, which responds to odor-stimulated electrical activity in OSNs by rapidly increasing transcription (Fischl et al., 2014), marks responding OSNs. Because each OSN expresses only one OR or TAAR, capturing fluorescent and nonfluorescent OSNs by FACS followed by expression profiling to quantify all mRNAs allows us to measure in a single experiment every OR and TAAR. The mRNAs encoding responsive ORs show significant shifts from $\mathrm{GFP}^{-}$samples to $\mathrm{GFP}^{+}$samples in odor-stimulated mice compared with control mice stimulated with clean air, a fact confirmed by in vitro experiments (McClintock et al., 2014; de March et al., 2020).

Consistent with our predictions about large numbers of receptors responsive to complex odors, such as cigarette smoke, we detect responses from 144 ORs and 3 TAARs in freely behaving mice exposed to cigarette smoke (Fig. 1A; Table 2). These responses are specific to cigarette smoke and are not observed in mice exposed only to clean air (Fig. $1 B$ ). Sequence relationships divide ORs into two groups: terrestrial vertebrate-specific Class II ORs and the more evolutionarily ancient Class I ORs (Glusman et al., 2000). The ORs responsive to cigarette smoke are nearly all Class II ORs, with just one responsive Class I OR, Olfr619. The 143 Class II ORs responsive to cigarette smoke are widely distributed across the Class II portion of the OR sequence distance tree (Fig. 1C). This response pattern is robust, rich in breadth, and not concentrated around a few related or strongly responsive ORs (Fig. 1C). Instead, the response pattern consists 

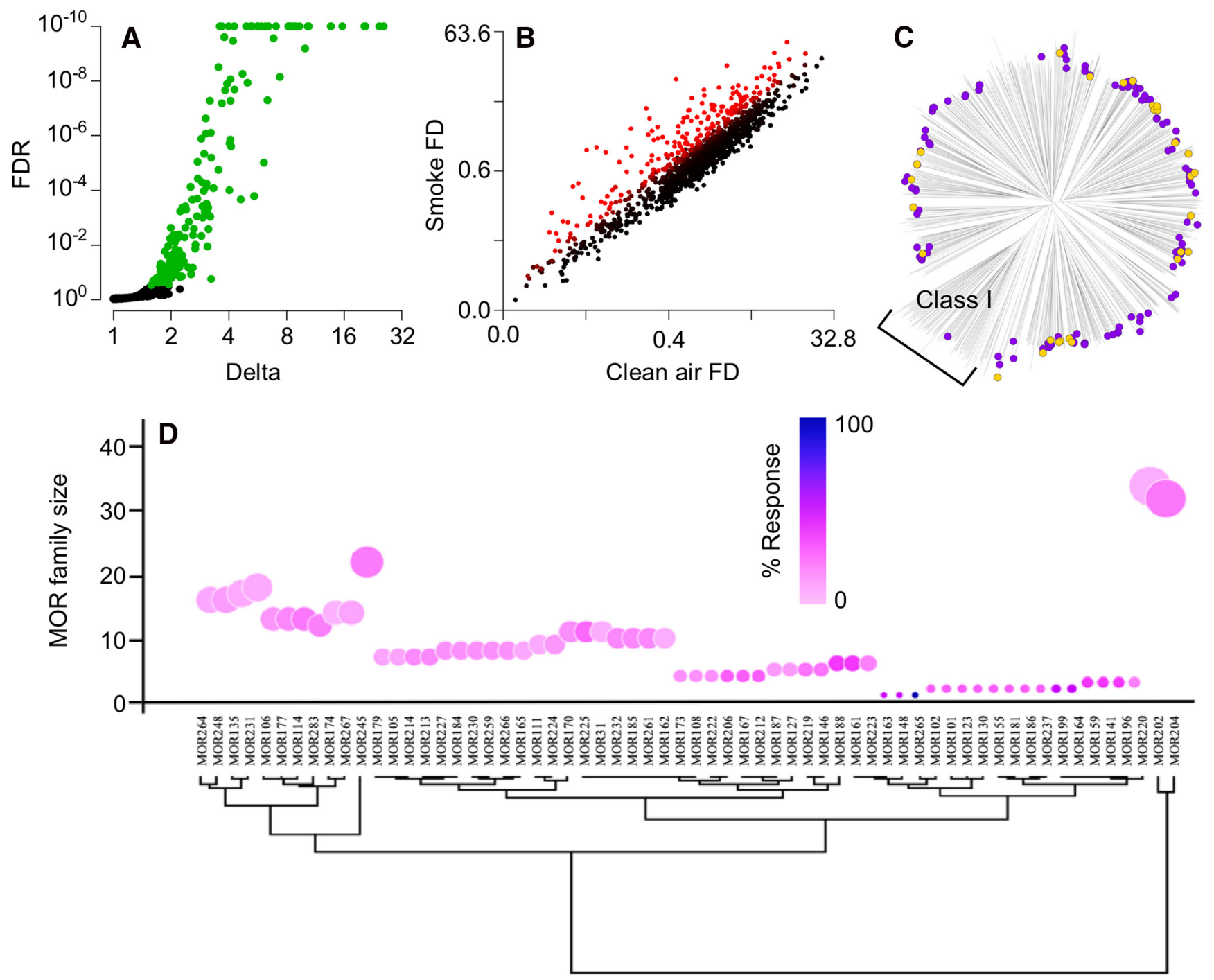

Figure 1. Receptors responsive to cigarette smoke in vivo. $\boldsymbol{A}$, In mice, $144 \mathrm{ORs}$ and 3 TAARs exceed the FDR criterion for a significant response to cigarette smoke. Delta is the cigarette smoke response relative to the clean air control response. FDR values capped at $10^{-10}$. B. The distribution of responses, measured as the fold difference (FD) enrichment of receptor mRNAs in samples of active OSNs compared with inactive OSNs, identifies responses specific to cigarette smoke versus the clean air control (red). C, Responsive ORs are widely distributed across the Class II portion of the OR sequence distance tree. Only 1 Class I OR responds. Gold represents responses $>5$-fold. $\boldsymbol{D}$, Clustering MOR families via similarity in family size and proportion of ORs responsive to cigarette smoke. No OR families contain large fractions of responsive $O R s$, and this fraction has little weight in the clustering of $O R$ families. Only $O R$ families containing responsive $O R$ s are shown. Circle size reflects family size.

of numerous peaks of strongly responsive receptors that are widely divergent in sequence. For example, the receptors whose response magnitude is fivefold or more include all three TAARs and 28 ORs that belong to 21 different OR families. Of the 186 Class II OR families in mice (Zhang and Firestein, 2002), 66 of them contain at least one responsive OR. Thirty-seven OR families have multiple responsive ORs, and families MOR204, MOR245, MOR114, and MOR225 contain five or more responsive ORs. However, these four families are all large, and the responsive ORs do not account for even a simple majority of ORs within them (Fig. 1D). The only instance where nearly all members of a multiple OR family are responsive to cigarette smoke is the MOR265 family, but it contains only two ORs (Fig. 1D).

The responsive TAARs include two closely related sequences, Taar7d and Taar7f, and the more distantly related Taar2. The odorant agonists of these three TAARs are not well defined, but TAAR7f is known to respond to N-methylpiperidine and N,Ndimethylcyclohexylamine, whereas TAAR7d also responds to $\mathrm{N}$,
N-dimethylcyclohexylamine (Liberles and Buck, 2006; Dewan et al., 2018). Of these two volatile amines, only $\mathrm{N}$-methylpiperidine is known to be present in cigarette smoke (Rodgman and Perfetti, 2013). TAAR2 is one of several TAARs believed to be more responsive to primary amines, whereas the TAAR7 family may be more responsive to tertiary amines (Ferrero et al., 2012).

\section{ORs highly sensitive to a mimic of cigarette smoke odor}

Perception of the odor of cigarette smoke does not require all of the $>400$ odorants found in cigarette smoke. Odors resembling cigarette smoke odor have been produced using many fewer odorants (Dravnieks et al., 1975; Cortese et al., 2015b). An effective mimic reported by Dravnieks et al. (1975) contains 32 odorants known to occur in cigarette smoke or closely related in structure to an odorant found in cigarette smoke, with 26 core odorants and 6 odorants suspected of arising from chemicals or flavorings added to cigarettes. We formulated a mimic containing the 26 core odorants. The receptors highly sensitive to this 
Table 2. Receptors responsive to cigarette smoke

\begin{tabular}{|c|c|c|c|c|c|c|c|c|}
\hline$\overline{\mathrm{OR}}$ & MOR name & Delta & OR & MOR name & Delta & OR & MOR name & $\overline{\text { Delta }}$ \\
\hline Olfr488 & MOR204-15 & 25.75 & Olfr1257 & MOR232-1 & 3.58 & $0 \mid$ lfr905 & MOR167-1 & 2.29 \\
\hline Olfr1278 & MOR245-11 & 24.46 & Olfr1135 & MOR177-2 & 3.53 & Olfr1014 & MOR213-5 & 2.28 \\
\hline Olfr1137 & MOR177-20 & 20.69 & Olfr1495 & MOR266-9 & 3.53 & Olfr433 & MOR123-1 & 2.23 \\
\hline $0 \mid$ lfr736 & MOR106-5 & 15.62 & Olfr1123 & MOR264-17 & 3.24 & Olfr1204 & MOR232-6 & 2.21 \\
\hline Olfr484 & MOR204-16 & 13.62 & Olfr1048 & MOR187-2 & 3.22 & Olfr1500 & MOR212-4P & 2.20 \\
\hline Taar2 & & 13.57 & Olfr1 & MOR135-13 & 3.20 & Olfr20 & MOR135-11 & 2.20 \\
\hline Olfr1277 & MOR248-11 & 9.97 & Olfr1026 & MOR196-4 & 3.12 & Olfr930 & MOR171-46 & 2.16 \\
\hline Olfr153 & MOR177-5 & 9.32 & Olfr1044 & MOR185-4 & 3.09 & $01 f r 746$ & MOR106-12 & 2.16 \\
\hline Olfr894 & MOR170-5 & 8.90 & Olfr1131 & MOR177-4 & 3.09 & $0 \mid f r 809$ & M0R108-4 & 2.16 \\
\hline Olfr1047 & MOR188-3 & 8.57 & $01 f r 352$ & MOR136-10 & 3.09 & $0 \mid$ lfr876 & MOR161-1 & 2.14 \\
\hline Olfr1313 & MOR245-23 & 8.36 & $01 f r 976$ & MOR224-10 & 3.06 & $0 \mid \mathrm{fr} 3$ & MOR136-14 & 2.13 \\
\hline Olfr16 & MOR267-13 & 8.22 & Olfr479 & MOR267-15 & 3.06 & Olfr870 & MOR141-1 & 2.11 \\
\hline $0 \mid$ lfr360 & MOR159-1 & 6.42 & Olfr1198 & MOR225-13 & 2.97 & $0 \mid f r 482$ & MOR204-14 & 2.06 \\
\hline Olfr1161 & MOR174-2 & 6.35 & Olfr1033 & MOR199-2 & 2.95 & Olfr1097 & MOR206-2 & 2.05 \\
\hline Olfr1056 & M0R186-2 & 6.20 & Olfr860 & MOR146-2 & 2.93 & Olfr851 & MOR155-1 & 2.05 \\
\hline Olfr769 & MOR114-14 & 6.08 & Olfr899 & MOR170-8 & 2.91 & Olfr1306 & MOR245-15 & 2.04 \\
\hline $0 \mid f r 247$ & MOR265-1 & 5.88 & Olfr881 & MOR162-7 & 2.90 & Olfr509 & MOR267-14 & 2.01 \\
\hline Olfr855 & MOR148-1 & 5.66 & Olfr506 & MOR204-23 & 2.87 & Olfr1396 & MOR276-2 & 1.99 \\
\hline Taar7d & & 5.64 & Olfr918 & MOR164-3 & 2.87 & $0 \mid f r 486$ & MOR204-19 & 1.99 \\
\hline Olfr1032 & MOR199-1 & 5.61 & Olfr295 & MOR220-1 & 2.76 & Olfr481 & MOR204-2 & 1.98 \\
\hline Olfr1182 & MOR225-7P & 5.41 & Olfr1316 & MOR245-1 & 2.75 & Olfr1254 & MOR231-13 & 1.98 \\
\hline Olfr1090 & MOR188-4 & 5.26 & Olfr $1206^{b}$ & MOR230-3 & 2.74 & Olfrgo8 & MOR165-2 & 1.98 \\
\hline Taar7f & & 5.19 & Olfr $176^{b}$ & MOR184-8 & 2.73 & Olfr1325 & MOR102-1 & 1.98 \\
\hline Olfr1280 & MOR248-1 & 5.00 & Olfr1303 & MOR245-7 & 2.68 & Olfr1338 & MOR259-9 & 1.97 \\
\hline Olfr1489 & MOR202-19 & 4.07 & Olfr1030 & MOR196-2 & 2.46 & $01 f r 312$ & MOR222-4P & 1.86 \\
\hline Olfr1122 & MOR264-1 & 4.07 & Olfr294 & MOR219-5 & 2.39 & $0 \mid$ lfr780 & MOR114-12 & 1.84 \\
\hline Olfr1140 & MOR177-6 & 4.03 & Olfr1243 & MOR231-4 & 2.37 & Olfr924 & MOR171-47 & 1.80 \\
\hline $0 \mid$ lfr467 & MOR204-33P & 4.02 & Olfr1434 & MOR214-4 & 2.37 & $01 f r 935$ & MOR171-11 & 1.79 \\
\hline Olfr819 & MOR265-2_p & 3.99 & 01 lfr738 & MOR106-3 & 2.35 & Olfr141 & MOR179-5 & 1.78 \\
\hline $0 \mid$ lfr706 & MOR283-11 & 3.91 & Olfr1201 & MOR230-2 & 2.33 & $01 f r 859$ & MOR146-3 & 1.77 \\
\hline Olfr1307 & MOR245-19P & 3.82 & Olfr803 & MOR111-3 & 2.33 & Olfr705 & MOR283-2 & 1.77 \\
\hline Olfr1366 & MOR130-2 & 3.78 & Olfr1281 & MOR248-18 & 2.30 & Olfr693 & MOR283-8 & 1.76 \\
\hline Olfr1272 & MOR227-3 & 3.68 & Olfr771 & M0R114-8 & 2.29 & Olfr444 & MOR261-2 & 1.76 \\
\hline Olfr521 & MOR101-2 & 3.67 & Olfr835 & MOR219-5 & 2.29 & Olfr470 & MOR204-22 & 1.75 \\
\hline
\end{tabular}

${ }^{a}$ Also responsive to artificial cigarette smoke odor and 1-pentanethiol.

${ }^{b}$ Also responsive to 1-pentanethiol.

'Also responsive to artificial cigarette smoke odor.

artificial cigarette smoke odor should represent receptors critical for forming a response pattern that is perceived by the brain as similar to the odor of cigarette smoke. Identifying these highly sensitive receptors would be complicated if some ORs have nonmonotonic dose-response relationships with their odorant agonists, a phenomenon predicted by observations of olfactory bulb glomeruli dropping out of glomerular response patterns as odorant concentration increases and confirmed by measures of OR responses in vivo (Friedrich and Korsching, 1997; Rubin and Katz, 1999; Meister and Bonhoeffer, 2001; Wachowiak and Cohen, 2001; Fried et al., 2002; Bozza et al., 2004; Hu et al., 2020; McClintock et al., 2020). To ensure that we identified sensitive
ORs, we therefore tested artificial cigarette smoke odor under conditions where the odor concentration reaching the mice was substantially less than saturation. Under these conditions, four ORs gave significant responses (Fig. 2A). These responses were specific to artificial cigarette smoke odor and did not occur when the mice were exposed to clean air (Fig. 2B). Three of these ORs, Olfr1202, Olfr1204, and Olfr1257, belong to the MOR232 family, while Olfr705 belongs to the MOR283 family (Fig. 2C). These two OR families have 10 or more members, and both have multiple members responsive to real cigarette smoke, including all four ORs responsive to artificial cigarette smoke odor (Fig. 2D). 


\section{1-Pentanethiol is important for the perception of artificial cigarette smoke odor}

To determine which odorants matter most to the artificial mimic of cigarette smoke odor, we asked human volunteers to rate the similarity of artificial cigarette smoke odor to 26 mixtures, each lacking one of the 26 odorants in the full mixture. Only two of these depletion mixtures prove to be perceived by human subjects as significantly different from the full mixture. Mixtures lacking 1-pentanethiol and 2methoxyphenol are perceived as different from artificial cigarette smoke odor and from the depletion mixture most similar to artificial cigarette smoke odor (Fig. 3).

The importance of 1-pentanethiol and 2-methoxyphenol for the percept evoked by artificial cigarette smoke odor could arise via either of two very different mechanisms. The most straightforward mechanism is that these odorants evoke responses from ORs important to the cigarette smoke response pattern, and therefore by themselves evoke percepts resembling that of artificial cigarette smoke odor. Alternatively, many odorants are not only agonists at some ORs but are also antagonists at other ORs (Araneda et al., 2000; Spehr et al., 2003; Oka et al., 2004a,b; Sanz et al., 2005, 2008; Reisert, 2010), a phenomenon that is common in vivo (de March et al., 2020). Such antagonist effects could be critical for the perception of odor mixtures because of their ability to substantially alter the OR response pattern. To discriminate between these mechanisms, we asked human volunteers to rate the similarity and pleasantness of 14 individual odorants to artificial cigarette smoke odor. Odorants whose percepts resemble that of artificial cigarette smoke odor are unlikely to be important to the perception of cigarette smoke odor solely through receptor antagonism. The 14 odorants included 11 odorants present in the artificial cigarette smoke odor mixture, including the four odorants whose absence most affected perception of artificial cigarette smoke odor (1-pentanethiol, 2methoxyphenol, 2-ethyl-3-methylpyrazine, and 5-methylfurfural) and seven odorants whose absence had little effect on the perception of artificial cigarette smoke odor (2,4-hexedienal, methyl isonicotinate, pyridine, 2-methylphenol, allylamine, indene, and diacetyl). Other odorants were included to act as outliers (acetophenone, DL-limonene, and 2-menthene). These experiments identify 1-pentanethiol, 2ethyl-3-methylpyrazine, allylamine, and indene as having properties expected of an odorant acting as an important
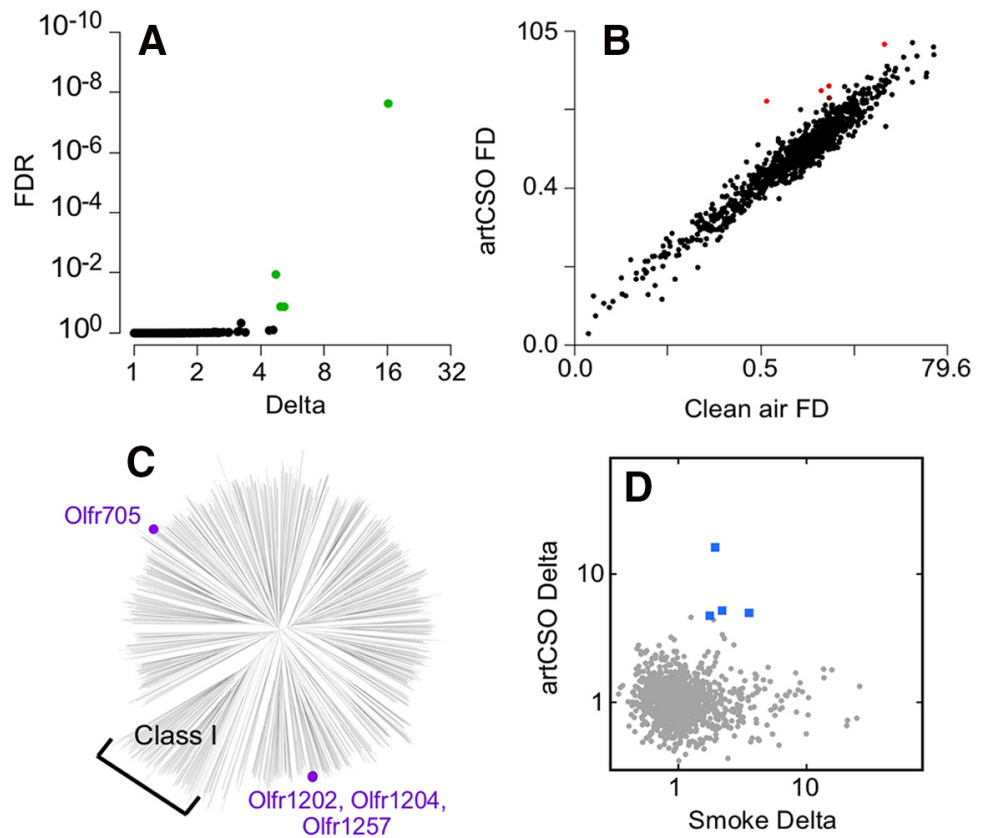

Figure 2. ORs responsive to artificial cigarette smoke in vivo. $\boldsymbol{A}$, In mice exposed to a low concentration of artificial cigarette smoke odor (artCSO), only four receptors respond, and they are all ORs. Delta is the artificial cigarette smoke odor response relative to the clean air control response. $\boldsymbol{B}$, The distribution of responses, measured as the fold difference (FD) enrichment of receptor mRNAs in samples of active OSNs compared with inactive OSNs, identifies responses specific to artificial cigarette smoke odor versus the clean air control (red). C, Of the responsive ORs, three are closely related to each other and not related to Olfr705, the other responsive OR. D, All four of the ORs responsive to a low concentration of artificial cigarette smoke odor (artCSO) also respond to cigarette smoke (blue squares).
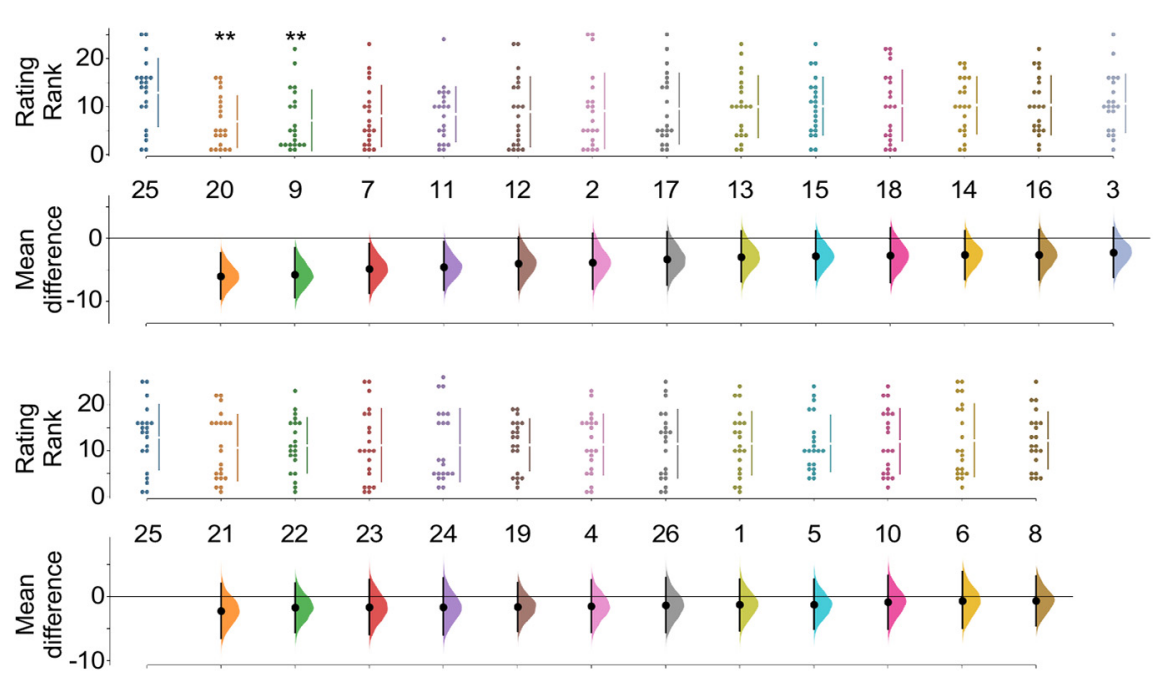

Figure 3. 1-Pentanethiol and 2-methoxyphenol matter most. Subjects $(n=18)$ rated 26 odor mixtures, each lacking one of the components of an artificial mimic of cigarette smoke odor, scoring the degree of similarity to the full mixture. The depleted mixture most similar to the full mixture lacks 2,4-hexedienal (\#25), and only the mixtures lacking 1-pentanethiol (\#20) and 2-methoxyphenol (\#9) are significantly different from it. 0dorants: 1, pyridine $(t=0.60, p=0.553) ; 2$, nicotinaldehyde $(t=1.65, p=0.101) ; 3$, 5-ethyl-2-methylpyridine $(t=1.10, p=0.278) ; 4,2$-vinylpyridine $(t=0.72, p=0.474) ; 5,4$ pyridinecarbonitrile $(t=0.63, p=532) ; 6$, methyl isonicotinate $(t=0.29, p=775) ; 7$, 2-ethyl-3-methylpyrazine $(t=2.34$, $p=0.024) ; 8,2$-methoxy-3,5-methylpyrazine $(t=0.32, p=0.747) ; 9,2$-methoxyphenol $(t=2.78, p=0.008) ; 10,2$-methylphenol $(t=0.39, p=0.696) ; 11,5$-methylfurfural $(t=2.28, p=0.028) ; 12,2,5$-dimethylpyrrole $(t=1.81, p=0.078) ; 13$, phenylacetylene $(t=1.41, p=0.166) ; 14$, isoprene $(t=1.30, p=0.201) ; 15,1,3$-cyclohexadiene $(t=1.38, p=174) ; 16$, indene $(t=1.28, p=0.207) ; 17$, allylbenzene $(t=1.50, p=142) ; 18$, diacetyl $(t=1.21, p=0.231) ; 19$, thiophene $(t=0.82$, $p=416)$; 20, 1-pentanethiol $(t=3.09, p=0.004) ; 21$, allylamine $(t=1.01, p=0.316) ; 22$, 1-aminopentane $(t=0.85$, $p=0.402) ; 23$, butyric acid $(t=0.72, p=0.475) ; 24$, 1-hexanal $(t=0.72, p=0.477) ; 25,2,4$-hexedienal; 26, indole $(t=0.62, p=0.541)$. ${ }^{* *} p<0.01$. 


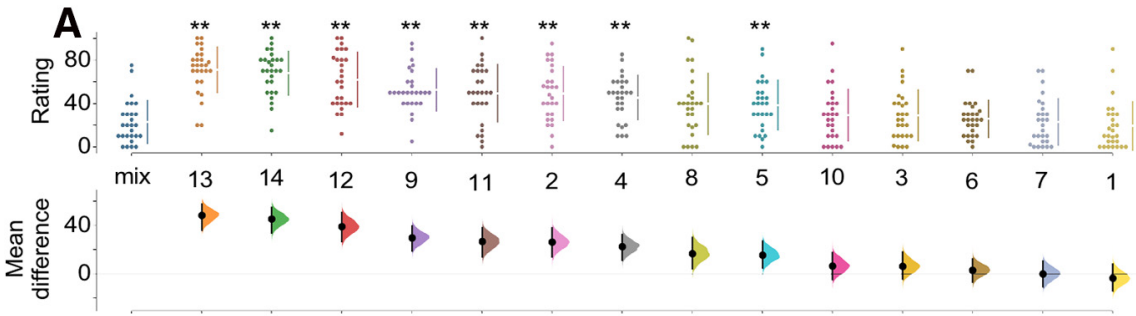

Pleasantness

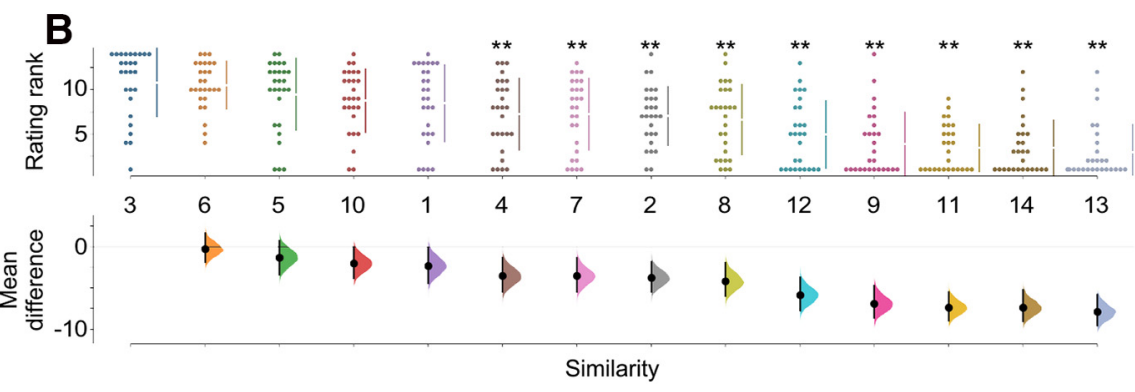

Figure 4. Odorants similar to cigarette smoke include 1-pentanethiol. $\boldsymbol{A}$, Subjects $(n=27)$ rated the pleasantness of 14 monomolecular odorants, finding that only six of these odorants were not significantly different from artificial cigarette smoke odor (mix). $\boldsymbol{B}$, Four of these six odorants also failed to be dissimilar from artificial cigarette smoke odor. Odorants: 1 , 1-pentanethiol (pleasantness $t=0.61, p=0.538$; similarity $t=2.10, p=0.040)$; 2, 2-methoxyphenol $(t=-4.30, p=0.000$; $t=3.88, p=0.000) ; 3$, 2-ethyl-3-methylpyrazine $(t=-1.07, p=0.291) ; 4,5$-methylfurfural $(t=-4.10, p=0.000) ; 5$, diacetyl $(t=-2.68, p=0.009 ; t=1.25, p=0.218) ; 6$, allylamine $(t=-0.61, p=0.542 ; t=0.33, p=0.744) ; 7$, pyridine $(t=-0.01, p=0.990 ; t=3.33, p=0.0 .002) ; 8,2$-methylphenol $(t=-2.54, p=0.015 ; t=3.95, p=0.000) ; 9$, methyl isonicotinate $(t=-5.59, p=0.000 ; t=6.86, p=0.000) ; 10$, indene $(t=-1.08, p=0.287 ; t=2.02, p=0.048) ; 11,2,4$-hexedienal $(t=-4.21, p=0.000 ; t=8.24, p=0.000) ; 12$, acetophenone $(t=-6.36, p=0.000 ; t=5.64, p=0.000) ; 13$, DL-limonene $(t=-8.71, p=0.000 ; t=8.31, p=0.000) ; 14$, 2-menthene $(t=-8.34, p=0.000 ; t=7.78, p=0.000)$. ${ }^{* *} p<0.01$

agonist in artificial cigarette smoke odor. They are not significantly different from artificial cigarette smoke odor in terms of perceived similarity or in terms of perceived pleasantness (Fig. 4). Overall, 1-pentanethiol is the only odorant whose absence has a significant effect on the perception of artificial cigarette smoke and also is perceived by itself as similar to cigarette smoke odor by humans.

\section{ORs responsive to 1-pentanethiol include ORs responsive to cigarette smoke}

If 1-pentanethiol is truly important for the perception of cigarette odors, both real cigarette smoke and artificial mimics, we should find that several ORs responsive to it are among the ORs responsive to cigarette smoke. To test this hypothesis, we exposed freely behaving mice to headspace air above a solution of 25\% 1-pentanethiol. This dilution exposes the mice to a relatively high, but not saturating, concentration of 1-pentanethiol and should result in responses from a substantial number of ORs. This prediction proved correct, with 58 ORs responding to 1-pentanethiol and not to the clean air control (Fig. $5 A, B$; Table 3). Of the 58 responsive ORs, 9 also respond to cigarette smoke: Olfr176, Olfr491, Olfr1137, Olfr1189, Olfr1202, Olfr1204, Olfr1206, Olfr1280, and Olfr1396. Of these, Olfr1137 is of particular interest because its response to cigarette smoke is the third largest we observed. In addition, both Olfr1202 and Olfr1204 also respond to artificial cigarette smoke odor.

The ORs responsive to 1-pentanethiol are distributed in clusters across the OR sequence distance tree, with 28 OR families containing at least 1 responsive OR and 13 OR families containing multiple responsive ORs (Fig. 5D). This response pattern is less widely distributed than that of cigarette smoke, and in certain OR families a large fraction of their ORs respond to 1-pentanethiol (Fig. 5E). In particular, a majority of ORs in families MOR230, MOR275, and MOR234 are responsive. This finding suggests that the ORs in these families might be particularly sensitive to thiols, or perhaps more broadly sensitive to short chain odorants.

Among the mouse ORs responsive to 1-pentanethiol are several that are common to receptor response patterns to odors that give rise to percepts related to cigarette smoke in humans. For example, the four ORs highly sensitive to artificial cigarette smoke odor are all part of the response pattern to real cigarette smoke and the response pattern to 1-pentanethiol contains 9 ORs responsive to real cigarette smoke. These overlaps in responsive ORs reflect a degree of similarity in the overall OR response patterns for artificial cigarette smoke odor, 1-pentanethiol, and real cigarette smoke. To assess the importance of these degrees of similarity, we compared response magnitudes measured for all ORs and TAARS in the in vivo assay for nine different odors: the three odors tested herein and six odors whose responses we published previously (Fig. 6). As expected, the patterns of response magnitude are significantly similar between 1-pentanethiol and artificial cigarette smoke odor, consistent with the finding that human subjects judge 1-pentanethiol to be the odorant component most important for perception of artificial cigarette smoke odor. Significantly similar pairs of response patterns also include the response patterns to concentrations of the same odor, a three-odorant mixture called a citrus accord, and the response patterns of two aldehydes, bourgeonal and undecanal. However, neither the 1-pentanethiol response pattern nor the artificial cigarette smoke odor response pattern is significantly similar to the response pattern to real cigarette smoke. These results suggest that the perception of two odors can in some cases be similar despite having large differences in OR response patterns.

\section{Discussion}

The data obtained in this project, when interpreted in the context of our prior understanding of the initial encoding of odor signals, support five conclusions. (1) ORs responsive to 1-pentanethiol are important elements of the pattern of ORs responsive to cigarette smoke and related odors. In mice, support for this assertion is found in the ORs whose responses are shared across 1-pentanethiol, artificial cigarette smoke odor, and real cigarette smoke. In humans, support is found in the impact of removing 1-pentanethiol from artificial cigarette smoke odor and the similarity in perception between artificial cigarette smoke odor and 1-pentanethiol. (2) The cigarette smoke receptor response pattern is robust and broad. Even the 28 ORs most strongly responsive to it are widely divergent sequences distributed across 21 OR families. (3) The cigarette smoke OR response pattern is the first in vivo response pattern observed to include both ORs and TAARs, consistent with the presence of odorants containing amine groups among the $>400$ odorants found in cigarette smoke (Rodgman 

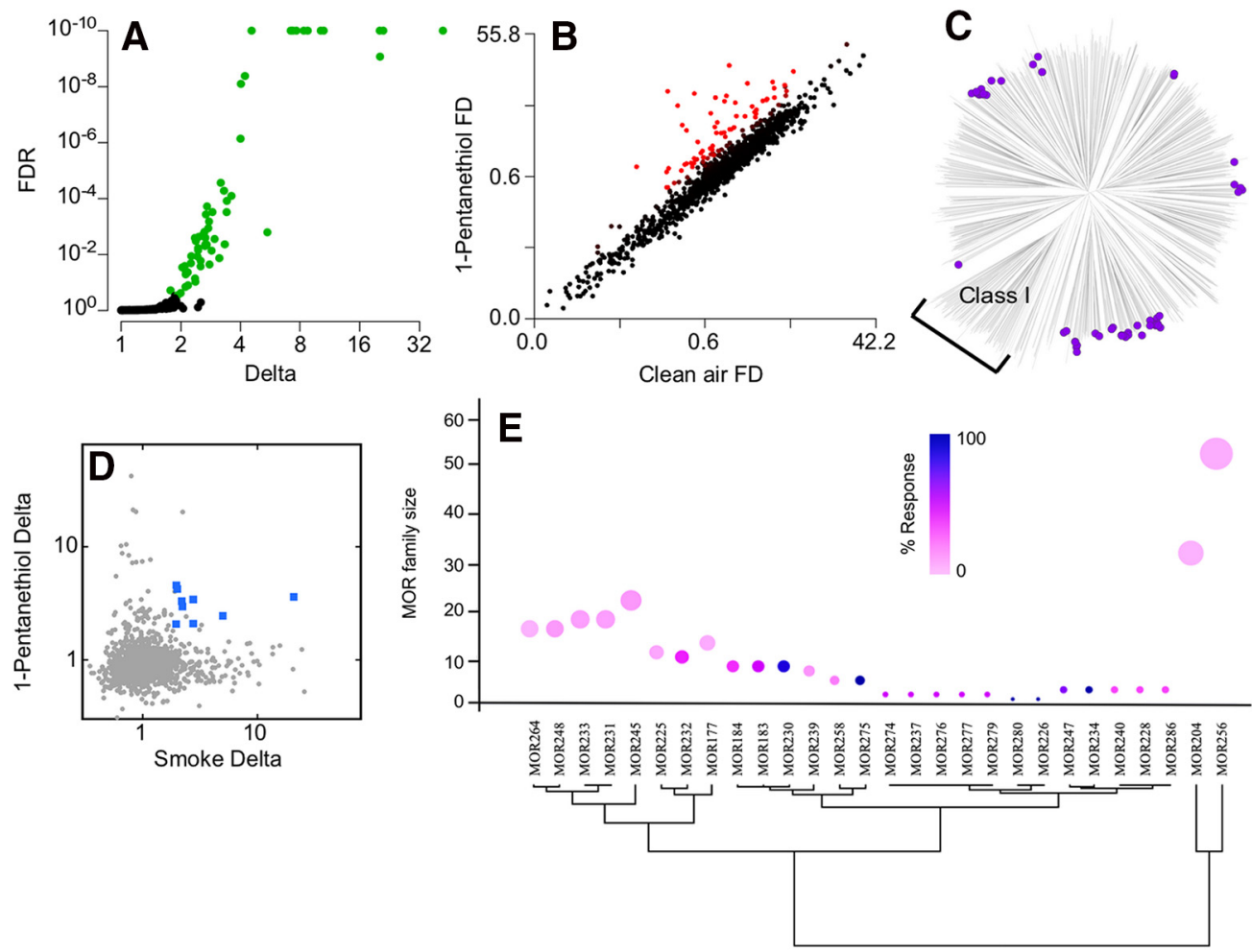

Figure 5. ORs responsive to 1-pentanethiol in vivo. $\boldsymbol{A}$, In mice, $58 \mathrm{ORs}$ respond to headspace air above a solution of 25\% 1-pentanethiol. Delta is the 1-pentanethiol response relative to the clean air control response. FDR values capped at $10^{-10}$. B . The distribution of responses, measured as the fold difference (FD) enrichment of receptor mRNAs in samples of active 0SNs compared with inactive OSNs, identifies responses specific to 1-pentanethiol versus the clean air control. C, The responsive ORs are distributed in several clusters across the Class II portion of the OR sequence distance tree. $\boldsymbol{D}$, of the responsive ORs, nine are also responsive to cigarette smoke (blue squares). $\boldsymbol{E}$, Clustering MOR families via similarity in family size and proportion of ORs responsive to 1-pentanethiol. Only a few OR families contain large fractions of responsive ORs. Only OR families containing responsive ORs are shown. Circle size reflects family size.

Table 3. ORs responsive to 1-pentanethiol

\begin{tabular}{|c|c|c|c|c|c|c|c|c|}
\hline$\overline{\mathrm{OR}}$ & MOR name & Delta & OR & MOR name & Delta & OR & MOR name & $\overline{\text { Delta }}$ \\
\hline $01 \mathrm{fr} 328$ & MOR275-2 & 41.90 & Olfr1261 & MOR234-3 & 3.34 & Olfr $1280^{b}$ & MOR248-1 & 2.46 \\
\hline Olfr195 & MOR184-5 & 21.08 & Olfr $491^{b}$ & MOR204-11 & 3.31 & Olfr1232 & MOR233-18 & 2.44 \\
\hline $0 \mid$ ffr224 & MOR275-3 & 20.31 & 0lfr329-ps & M0R275-6P & 3.19 & Olfr1299 & MOR248-8 & 2.44 \\
\hline Olfr1183 & MOR230-6 & 20.25 & Olfr124 & MOR256-3 & 3.13 & Olfr1256 & MOR231-1 & 2.43 \\
\hline $0 \mid$ lfr330 & MOR275-1 & 10.52 & Olfr1204 & MOR232-6 & 2.97 & Olfr1427 & MOR239-4 & 2.38 \\
\hline Olfr1193 & MOR226-1 & 10.19 & Olfr31 & MOR274-1 & 2.89 & Olfr192 & MOR183-x & 2.37 \\
\hline Olfr1395 & MOR277-1 & 8.76 & Olfr1195 & MOR230-4 & 2.86 & $0 \mid f r 331$ & MOR275-4 & 2.36 \\
\hline $01 \mathrm{fr} 325$ & MOR275-5 & 8.38 & Olff48 & MOR232-5 & 2.80 & Olfr1262 & MOR234-1 & 2.35 \\
\hline Olfr1279 & MOR245-12 & 7.69 & Olfr193 & MOR183-7P & 2.78 & Olfr1260 & M0R232-2 & 2.26 \\
\hline 01 lfr286 & MOR286-2 & 7.39 & 0lfr183 & M0R183-2 & 2.76 & Olfr1209 & MOR230-7 & 2.25 \\
\hline Olfr1284 & MOR245-13 & 7.29 & 0lfr723 & MOR247-4 & 2.71 & Olfr1288 & MOR245-9 & 2.17 \\
\hline Olfr1212 & MOR233-17 & 7.19 & Olfr165 & MOR279-1 & 2.71 & Olfr1321 & MOR264-26 & 2.16 \\
\hline Olfr1394 & MOR280-1 & 5.46 & Olfr191 & MOR183-5P & 2.68 & $0 \mid \mathrm{lfr} 724$ & MOR247-2 & 2.12 \\
\hline Olfr1189 & MOR237-2 & 4.55 & Olfr1263 & MOR234-2 & 2.66 & Olfr $176^{b}$ & MOR184-8 & 2.11 \\
\hline Olfr $1396^{b}$ & MOR276-2 & 4.21 & Olfr1342 & MOR258-3 & 2.66 & Olfr15 & MOR256-17 & 2.11 \\
\hline Olfr175-ps1 & MOR184-1 & 4.02 & Olfr1359 & MOR256-35 & 2.65 & Olfr $1202^{a}$ & M0R232-7 & 2.09 \\
\hline Olfr1186 & MOR230-5 & 4.00 & Olfr1265 & MOR228-2 & 2.63 & $0 \mid$ lfr464 & MOR240-2 & 2.08 \\
\hline Olfr1137 & MOR177-20 & 3.60 & Olfr1200 & MOR225-12 & 2.63 & Olfr1242 & MOR231-5 & 2.03 \\
\hline Olfr $1206^{b}$ & MOR230-3 & 3.42 & $0 \mid f r 476$ & MOR204-3 & 2.58 & & & \\
\hline Olfr1199 & MOR230-8 & 3.41 & Olfr1205 & MOR230-1 & 2.52 & & & \\
\hline
\end{tabular}

${ }^{a}$ Also responsive to cigarette smoke and artificial cigarette smoke odor.

${ }^{b}$ Also responsive to cigarette smoke.

and Perfetti, 2013). (4) Class I ORs are not important to the perception of cigarette smoke. Class II ORs constitute all but one of the ORs responsive to cigarette smoke and all of the ORs responsive to 1-pentanethiol and artificial cigarette smoke odor. (5) Our data provide the first example of odors evoking similar percepts while sharing ORs in their in vivo response patterns. This is an anticipated result, but we are not confident this correlation will always prove true and urge caution before presuming that it does.

1-Pentanethiol is important to the perception of odors related to cigarette smoke, and ORs responsive to it are part of the OR response pattern to cigarette smoke and artificial cigarette smoke 


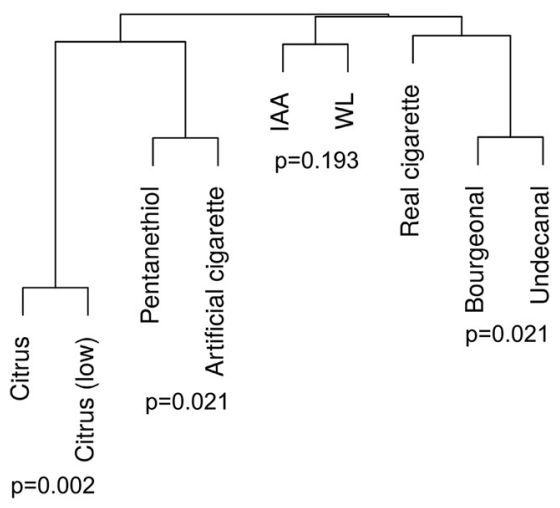

Figure 6. Response pattern similarity. Cluster analysis displays relative similarity between in vivo receptor response patterns to nine odors tested in this study or published previously (de March et al., 2020; McClintock et al., 2020). Statistical analysis identifies three significantly similar response patterns, including that of 1-pentanethiol (Pentanethiol) and artificial cigarette smoke odor (Artificial cigarette). The receptor response pattern to cigarette smoke (Real cigarette) is not similar to any of the other response patterns. The citrus accord odors (Citrus), which are mixtures of three odorants, differ by 20 -fold in the concentrations used as stimuli ( $5 \%$ vs $100 \%)$.

odor. Our human testing data confirm that 1-pentanethiol evokes a percept similar to artificial cigarette smoke odor; and correspondingly, the mouse OR response patterns between these two odors are more similar than chance. However, ORs responsive to 1-pentanethiol explain only a small part of the receptor response to real cigarette smoke, a portion insufficient to produce significant similarity between the overall response patterns of 1-pentanethiol and real cigarette smoke. Not surprisingly, 1pentanethiol must be just one of several odorants important to the cigarette smoke response pattern. The identities of the others remain to be discovered.

The OR response pattern for 1-pentanethiol is also potentially indicative of ORs that respond to specific features of odorant molecules. This is especially true of families MOR230, MOR275, and MOR234 where a large fraction of the members of the family respond to 1-pentanethiol. Whether these groups of related ORs share sensitivity to certain simple thiols, or more broadly to many types of short chain odorants, pose interesting questions for future study. We do know that these are not the only families of ORs responsive to thiols. Olfr1509, a mouse OR responsive to a more complex thiol odorant, (methylthio)methanethiol (Duan et al., 2012), is not one of the ORs responsive to 1-pentanethiol, and other members of the same OR family (MOR244) also did not respond to 1-pentanethiol in our experiments.

Cigarette smoke is the first complex odor whose in vivo receptor response pattern has been measured. The breadth of the receptor response pattern evoked by cigarette smoke and the fact that the strongest responses are distributed across the diversity of Class II OR sequences and the TAARs are instructive. This is not simply because these complex odors contain so many species of odorants, although this is a contributing factor. The 1-pentanethiol OR response pattern by itself contains more than one-third of the number of receptors responsive to cigarette smoke and more than one-third of the number of OR families with members responsive to cigarette smoke. This breadth is consistent with previous findings that the response patterns of isoamyl acetate, bourgeonal, whiskey lactone, acetophenone, 2,5-dihydro2,4,5-trimethylthiazoline, and carvones include ORs from several unrelated OR families (Hamana et al., 2003; Jiang et al., 2015; de March et al., 2020). We conclude that this breadth of responsivity is a characteristic feature of OR response patterns, not only for complex odors but also for individual odorants. This conclusion is consistent with evidence that specific anosmias are relatively rare, even while hyposmias are fairly common and generally consist of reduced detection thresholds for certain odorants (Trimmer et al., 2019). Loss or mutation of the OR most sensitive to an odorant would lead to reduced sensitivity to the odorant, but not absence of detection, because other ORs less sensitive to the odorant will respond as concentration rises (Sato-Akuhara et al., 2016; Dewan et al., 2018). This understanding that most odorant responses consist of several unrelated ORs is also instructive about OR evolution. OR evolution must have been driven primarily by selective forces favoring expansion and diversification over forces selecting for ORs highly sensitive to, and specific for, individual odorants. This would allow OR evolution, and by extension the general olfaction function provided by the main olfactory epithelium, to keep pace with changing and expanding odor environments.

With $>400$ odorants, all thought to be capable of evoking responses from multiple receptors, why isn't the response to cigarette smoke even broader than what we observe? Some of this discrepancy is presumably because of some of the odorants in cigarette smoke being present at low concentrations incapable of producing responses detectable in the assay used. However, responses to mixtures of odorants have long been known to be less than the sum of the responses to the individual odorants in the mixture (Laing and Francis, 1989; Livermore and Laing, 1996; Poupon et al., 2018). Antagonism of ORs by odorants, which is very common in mixtures of odorants (de March et al., 2020), almost certainly also contributes. These odorant interactions at receptors result in response patterns that make discerning individual odorants in mixtures difficult and favor perceiving complex odors as distinct objects rather than a combination of the elements of the mixture (Thomas-Danguin et al., 2014). Cigarette smoke is certainly perceived as a distinct object rather than its component elements, and is one of the most common odors we humans encounter.

Odors that have similar percepts sufficient to belong to the same odor object categorization may do so because they share responses from some ORs. The OR responses shared between cigarette smoke, artificial cigarette smoke odor, and 1-pentanethiol support this hypothesis. Indeed, perceptual similarity is known to correlate with the degree of similarity in neural activity at multiple levels of the mammalian olfactory system, and this is expected to arise from similarity in OR response patterns (Wilson, 2009; Gottfried, 2010; Pashkovski et al., 2020). However, our evidence is not yet sufficient to have confidence that this hypothesis will prove correct for all cases of similar percepts, in part because our evidence is a correlation between human perception and mouse receptor physiology. In addition, we cannot rule out the possibility that cortical pattern completion processing mechanisms might sometimes find similarity between odors that do not have overlapping OR response patterns but instead have overlap at higher levels of olfactory signal processing. Furthermore, the inverse hypothesis of OR response pattern similarity necessarily resulting in perceptual similarity is almost certainly false. Instances of highly similar odorants that differ substantially in their percepts, although they evoke strongly overlapping patterns of activity in OSNs or olfactory bulb glomeruli, strongly predict that similar OR response patterns can give rise to distinct percepts (Linster et al., 2001; Hamana et al., 2003). A specific example revealed in our comparison of receptor response patterns is the similarity of mouse OR response patterns for bourgeonal and undecanal, which humans 
perceive as being quite distinct. Our findings support the idea that OR response pattern similarity is a basis for perceptual similarity, perhaps even when response pattern similarity is just a small fraction of responsive receptors, but with the caveat that the CNS is also capable of producing distinct percepts even from significantly overlapping OR response patterns.

\section{References}

Araneda RC, Kini AD, Firestein S (2000) The molecular receptive range of an odorant receptor. Nat Neurosci 3:1248-1255.

Balfour DJ (2015) The role of mesoaccumbens dopamine in nicotine dependence. Curr Top Behav Neurosci 24:55-98.

Bolding KA, Franks KM (2018) Recurrent cortical circuits implement concentration-invariant odor coding. Science 361:eaat6904.

Bozza T, McGann JP, Mombaerts P, Wachowiak M (2004) In vivo imaging of neuronal activity by targeted expression of a genetically encoded probe in the mouse. Neuron 42:9-21.

Buck L, Axel R (1991) A novel multigene family may encode odorant receptors: a molecular basis for odor recognition. Cell 65:175-187.

Chess A, Simon I, Cedar H, Axel R (1994) Allelic inactivation regulates olfactory receptor gene expression. Cell 78:823-834.

Corish P, Tyler-Smith C (1999) Attenuation of green fluorescent protein half-life in mammalian cells. Protein Eng 12:1035-1040.

Cortese BM, Uhde TW, LaRowe SD, Stein SV, Freeman WC, McClernon FJ, Brady KT, Hartwell KJ (2015a) Olfactory cue reactivity in nicotine-dependent adult smokers. Psychol Addict Behav 29:91-96.

Cortese BM, Uhde TW, Brady KT, McClernon FJ, Yang QX, Collins HR, LeMatty T, Hartwell KJ (2015b) The fMRI BOLD response to unisensory and multisensory smoking cues in nicotine-dependent adults. Psychiatry Res 234:321-327.

de March CA, Titlow WB, Sengoku T, Breheny P, Matsunami H, McClintock TS (2020) Modulation of the combinatorial code of odorant receptor response patterns in odorant mixtures. Mol Cell Neurosci 104:103469.

Dewan A, Cichy A, Zhang J, Miguel K, Feinstein P, Rinberg D, Bozza T (2018) Single olfactory receptors set odor detection thresholds. Nat Commun 9:2887.

Dravnieks A, O'Donnell A, Reilich HG (1975) Determination of odor components in tobacco smoke: design of mixture to simulate odor. ASHRAE Trans 81:200-212.

Duan X, Block E, Li Z, Connelly T, Zhang J, Huang Z, Su X, Pan Y, Wu L, Chi Q, Thomas S, Zhang S, Ma M, Matsunami H, Chen GQ, Zhuang H (2012) Crucial role of copper in detection of metal-coordinating odorants. Proc Natl Acad Sci USA 109:3492-3497.

Efron B (2008) Microarrays, empirical Bayes and the two-groups model. Statist Sci 23:1-22

Ferrero DM, Wacker D, Roque MA, Baldwin MW, Stevens RC, Liberles SD (2012) Agonists for 13 trace amine-associated receptors provide insight into the molecular basis of odor selectivity. ACS Chem Biol 7:1184-1189.

Fischl AM, Heron PM, Stromberg AJ, McClintock TS (2014) Activity-dependent genes in mouse olfactory sensory neurons. Chem Senses 39:439449.

Fried HU, Fuss SH, Korsching SI (2002) Selective imaging of presynaptic activity in the mouse olfactory bulb shows concentration and structure dependence of odor responses in identified glomeruli. Proc Natl Acad Sci USA 99:3222-3227.

Friedrich RW, Korsching SI (1997) Combinatorial and chemotopic odorant coding in the zebrafish olfactory bulb visualized by optical imaging. Neuron 18:737-752.

Glusman G, Bahar A, Sharon D, Pilpel Y, White J, Lancet D (2000) The olfactory receptor gene superfamily: data mining, classification, and nomenclature. Mamm Genome 11:1016-1023.

Gottfried JA (2010) Central mechanisms of odour object perception. Nat Rev Neurosci 11:628-641.

Hajek P, Phillips-Waller A, Przulj D, Pesola F, Myers Smith K, Bisal N, Li J, Parrott S, Sasieni P, Dawkins L, Ross L, Goniewicz M, Wu Q, McRobbie HJ (2019) A randomized trial of e-cigarettes versus nicotine-replacement therapy. N Engl J Med 380:629-637.

Halpern SD, Harhay MO, Saulsgiver K, Brophy C, Troxel AB, Volpp KG (2018) A pragmatic trial of e-cigarettes, incentives, and drugs for smoking cessation. N Engl J Med 378:2302-2310.
Hamana H, Hirono J, Kizumi M, Sato T (2003) Sensitivity-dependent hierarchical receptor codes for odors. Chem Senses 28:87-104.

Hu XS, Ikegami K, Vihani A, Zhu KW, Zapata M, de March CA, Do M, Vaidya N, Kucera G, Bock C, Jiang Y, Yohda M, Matsunami H (2020) Concentration-dependent recruitment of mammalian odorant receptors. eNeuro 7:ENEURO.0103-19.2019.

Jiang Y, Gong NN, Hu XS, Ni MJ, Pasi R, Matsunami H (2015) Molecular profiling of activated olfactory neurons identifies odorant receptors for odors in vivo. Nat Neurosci 18:1446-1454.

Johnson MA, Tsai L, Roy DS, Valenzuela DH, Mosley C, Magklara A, Lomvardas S, Liberles SD, Barnea G (2012) Neurons expressing trace amine-associated receptors project to discrete glomeruli and constitute an olfactory subsystem. Proc Natl Acad Sci USA 109:13410-13415.

Keller A, Vosshall LB (2016) Olfactory perception of chemically diverse molecules. BMC Neurosci 17:55.

Laing DG, Francis GW (1989) The capacity of humans to identify odors in mixtures. Physiol Behav 46:809-814.

Liberles SD (2015) Trace amine-associated receptors: ligands, neural circuits, and behaviors. Curr Opin Neurobiol 34:1-7.

Liberles SD, Buck LB (2006) A second class of chemosensory receptors in the olfactory epithelium. Nature 442:645-650.

Linster C, Johnson BA, Yue E, Morse A, Xu Z, Hingco EE, Choi Y, Choi M, Messiha A, Leon M (2001) Perceptual correlates of neural representations evoked by odorant enantiomers. J Neurosci 21:9837-9843.

Livermore A, Laing DG (1996) Influence of training and experience on the perception of multicomponent odor mixtures. J Exp Psychol Hum Percept Perform 22:267-277.

Malnic B, Hirono J, Sato T, Buck LB (1999) Combinatorial receptor codes for odors. Cell 96:713-723.

McClintock TS, Wang Q, Sengoku T, Titlow WB, Breheny P (2020) Mixture and concentration effects on odorant receptor response patterns in vivo. Chem Senses Advance online publication. Retrieved May 19, 2020. doi: 10.1093/chemse/bjaa032.

McClintock TS, Adipietro K, Titlow WB, Breheny P, Walz A, Mombaerts P, Matsunami H (2014) In vivo identification of eugenol-responsive and muscone-responsive mouse odorant receptors. J Neurosci 34:1566915678 .

Meister M, Bonhoeffer T (2001) Tuning and topography in an odor map on the rat olfactory bulb. J Neurosci 21:1351-1360.

Mombaerts P (2004) Odorant receptor gene choice in olfactory sensory neurons: the one receptor-one neuron hypothesis revisited. Curr Opin Neurobiol 14:31-36.

Mombaerts P, Wang F, Dulac C, Chao SK, Nemes A, Mendelsohn M, Edmondson J, Axel R (1996) Visualizing an olfactory sensory map. Cell 87:675-686.

Nara K, Saraiva LR, Ye X, Buck LB (2011) A large-scale analysis of odor coding in the olfactory epithelium. J Neurosci 31:9179-9191.

Nickell MD, Breheny P, Stromberg AJ, McClintock TS (2012) Genomics of mature and immature olfactory sensory neurons. J Comp Neurol 520:2608-2609.

Niimura Y, Matsui A, Touhara K (2014) Extreme expansion of the olfactory receptor gene repertoire in African elephants and evolutionary dynamics of orthologous gene groups in 13 placental mammals. Genome Res 24:1485-1496.

Oka Y, Nakamura A, Watanabe H, Touhara K (2004a) An odorant derivative as an antagonist for an olfactory receptor. Chem Senses 29:815-822.

Oka Y, Omura M, Kataoka H, Touhara K (2004b) Olfactory receptor antagonism between odorants. EMBO J 23:120-126.

Pashkovski SL, Iurilli G, Brann D, Chicharro D, Drummey K, Franks K, Panzeri S, Datta SR (2020) Structure and flexibility in cortical representations of odour space. Nature 583:253-258.

Picciotto MR, Mineur YS (2014) Molecules and circuits involved in nicotine addiction: the many faces of smoking. Neuropharmacology 76:545-553.

Poupon D, Fernandez P, Boisvert SA, Migneault-Bouchard C, Frasnelli J (2018) Can the identification of odorants within a mixture be trained? Chem Senses 43:721-726.

Reisert J (2010) Origin of basal activity in mammalian olfactory receptor neurons. J Gen Physiol 136:529-540.

Rodgman A, Perfetti TA (2013) The chemical components of tobacco and tobacco smoke, Ed 2. Boca Raton, FL: CRC.

Rubin BD, Katz LC (1999) Optical imaging of odorant representations in the mammalian olfactory bulb. Neuron 23:499-511. 
Sammeta N, Yu TT, Bose SC, McClintock TS (2007) Mouse olfactory sensory neurons express 10,000 genes. J Comp Neurol 502:1138-1156.

Sanz G, Schlegel C, Pernollet JC, Briand L (2005) Comparison of odorant specificity of two human olfactory receptors from different phylogenetic classes and evidence for antagonism. Chem Senses 30:69-80.

Sanz G, Thomas-Danguin T, el Hamdani H, Le Poupon C, Briand L, Pernollet JC, Guichard E, Tromelin A (2008) Relationships between molecular structure and perceived odor quality of ligands for a human olfactory receptor. Chem Senses 33:639-653.

Sato-Akuhara N, Horio N, Kato-Namba A, Yoshikawa K, Niimura Y, Ihara S, Shirasu M, Touhara K (2016) Ligand specificity and evolution of mammalian musk odor receptors: effect of single receptor deletion on odor detection. J Neurosci 36:4482-4491.

Spehr M, Gisselmann G, Poplawski A, Riffell JA, Wetzel CH, Zimmer RK, Hatt $\mathrm{H}$ (2003) Identification of a testicular odorant receptor mediating human sperm chemotaxis. Science 299:2054-2058.

Stephens M (2017) False discovery rates: a new deal. Biostatistics 18:275-294.

Storace DA, Cohen LB (2017) Measuring the olfactory bulb input-output transformation reveals a contribution to the perception of odorant concentration invariance. Nat Commun 8:81.

Tamura H, Hata Y, Chida M, Yamashita K (2008) Picking aroma character compounds in Citrus limon oils by using odor thresholds in aroma mixtures. In: Food Flavor: Chemistry, Sensory Evaluation, and Biological
Activity (Tamura H, Ebeler SE, Kubota K, Takeoka GR, eds), pp 229242: American Chemical Society.

Thomas-Danguin T, Sinding C, Romagny S, El Mountassir F, Atanasova B, Le Berre E, Le Bon AM, Coureaud G (2014) The perception of odor objects in everyday life: a review on the processing of odor mixtures. Front Psychol 5:504.

Trimmer C, Keller A, Murphy NR, Snyder LL, Willer JR, Nagai MH, Katsanis N, Vosshall LB, Matsunami H, Mainland JD (2019) Genetic variation across the human olfactory receptor repertoire alters odor perception. Proc Natl Acad Sci USA 116:9475-9480.

Wachowiak M, Cohen LB (2001) Representation of odorants by receptor neuron input to the mouse olfactory bulb. Neuron 32:723735.

Welch BL (1947) The generalisation of student's problems when several different population variances are involved. Biometrika 34:2835.

Wilson DA (2009) Pattern separation and completion in olfaction. Ann NY Acad Sci 1170:306-312.

Yu TT, McIntyre JC, Bose SC, Hardin D, Owen MC, McClintock TS (2005) Differentially expressed transcripts from phenotypically identified olfactory sensory neurons. J Comp Neurol 483:251-262.

Zhang X, Firestein S (2002) The olfactory receptor gene superfamily of the mouse. Nat Neurosci 5:124-133. 